\title{
Bulanık FUCOM Metodu ile Tedarikçi Değerlendirme Kriterlerinin A ğırlıklarının Belirlenmesi
}

\author{
Merve Ceren Taşkent ${ }^{1}$, Elif Kılıç Delice ${ }^{2 *}$ \\ ${ }^{1}$ Atatürk Üniversitesi,Mühendislik Fakültesi, Endüstri Mühendisliği Bölümü, Erzurum, Türkiye (ORCID ID 0000-0001-6508-5568), mctaskent@gmail.com \\ ${ }^{2}$ Atatürk Üniversitesi,Mühendislik Fakültesi, Endüstri Mühendisliği Bölümü, Erzurum, Türkiye(ORCID ID 0000-0002-3051-0496), elif.kdelice@atauni.edu.tr
}

(1st International Conference on Applied Engineering and Natural Sciences ICAENS 2021, November 1-3, 2021)

(DOI: $10.31590 /$ ejosat.1011756)

ATIF/REFERENCE: Taşkent, M.C.., Delice, E.K. (2021). Bulanık FUCOM Metodu ile Tedarikçi Değerlendirme Kriterlerinin Ağırlıklarının Belirlenmesi. Avrupa Bilim ve Teknoloji Dergisi, (28), 863-868.

\begin{abstract}
$\ddot{O} \mathbf{z}$
E-ticaret ile birlikte firmaların ulaşabileceği pazar alanı uluslararası alana taşınmış ve bu durum firmaları, rekabet ortamında başarılı olabilmek için çeşitli iyileştirme çalışmalarını gerçekleştirmeye itmiştir. Bu iyileştirme çalışmalarından bir tanesi tedarik zinciri ağı içerisinde yer alan ve tedarik zinciri sürecini başlatan tedarikçilerdir. Tedarikçiler ürünün kalitesini, üretim süresini, üretim maliyetini ve hatta nihai ürünün son müşteriye teslimine kadar olan süreci doğrudan etkilemektedir. Bu sebeple işletmeler için uygun tedarikçileri seçmek veya mevcut tedarikçilerin performansların değerlendirmek için çaba göstermektedirler. Tedarikçi seçim ve değerlendirmesi sürecinde kullanılacak kriterler ve bu kriterlerin önem ağılıklarının son derece etkilidir. Bu çalışmada, tedarikçi seçim sürecinde kriter ağırlıklarının belirlenmesi için bulanık Full Consistency Method (FUCOM) metodu kullanılmıştır. Literatürde genellikle karar vericilerin sübjektif değerlendirmelerine bağlı olarak Çok Kriterli Karar Verme (ÇKKV) yöntemleri ile uygun kriter ağırlıkları belirlenmekte ve kesin sonuçlar elde edilememektedir. Bu çalışmada ise Bulanık FUCOM metodu ile sübjektif değerlendirmeler kullanılarak optimal kriter ağırlıkları belirlenmiştir. Dekoratif taş sağlayıcısı bir firmanın mevcut tedarikçilerinin değerlendirilmesi üzerine bir uygulama çalışması yapılmıştır.
\end{abstract}

\section{Determination the Weight of Supplier Evaluation Criteria with Fuzzy FUCOM Method}

\begin{abstract}
With e-commerce, the market area that companies can reach has been moved to the international arena and this situation has pushed companies to carry out various improvement studies in order to be successful in the competitive environment. One of these improvement efforts is the suppliers that are in the supply chain network and start the supply chain process. Suppliers directly affect the quality of the product, production time, production cost and even the process until the delivery of the final product to the end customer. For this reason, they strive to select suitable suppliers for businesses or to evaluate the performance of existing suppliers. The criteria to be used in the supplier selection and evaluation process and the importance weights of these criteria are extremely effective. In this study, the fuzzy Full Consistency Method (FUCOM) method was used to determine the criterion weights in the supplier selection process. In the literature, appropriate criteria weights are determined by Multi-Criteria Decision Making (MCDM) methods, depending on the subjective evaluations of decision makers, and definite results cannot be obtained. In this study, the optimal criterion weights were determined by using the fuzzy FUCOM method and subjective evaluations. An application study was conducted on the evaluation of the existing suppliers of a decorative stone supplier company.
\end{abstract}

Keywords: Fuzzy FUCOM, Supplier Evaluation, Criteria Weighting.

\footnotetext{
"Sorumlu Yazar: elif.kdelice@atauni.edu.tr
} 


\section{Giriş}

Teknolojik gelişmeler ile birlikte istenilen ürün veya hizmete ulaşımın kolaylaşması küreselleşme kavramını ortaya koyarak firmaların yerel pazardan uluslararası pazara geçişini sağlamıştır. $\mathrm{Bu}$ şekilde firmalar ulaşabilecekleri maksimum müşteri kapasitenin dışına çıkarak uluslararası pazarda yer edinme için marka değerlerini iyileştirme çalışmalarına özen göstermişlerdir. $\mathrm{Bu}$ iyileştirme alanlarından bir tanesi tedarik zinciri yönetimidir. Tedarik zinciri yönetimi içerisindeki konular incelendiğinde bunların doğrudan tedarikçi ile ilişkili olduğu görülmektedir. Artan müşteri sayısı ile tedarikçi sayısı sonucu uygun tedarikçinin seçimi veya mevcut tedarikçilerinin performansının değerlendirilmesi firmalar için önemli bir karar problemi olarak görülmektedir [33].

Tedarikçi seçim veya değerlendirme süreci yalnızca en iyi fiyat ile en iyi teslimatı sunan tedarikçiyi bulmanın ötesinde, optimal faydayı sağlayacak tedarikçiyi kapsamaktadır. Optimal fayda değeri tedarikçi seçiminde tedarikçinin performansının ölçüsüdür ve bu değeri etkileyen birçok unsur bulunmaktadır. $\mathrm{Bu}$ unsurlara teslimat performansı, geçmiş performansı, garanti ve sistem politikaları, üretim tesisleri ve kapasitesi, fiyat, teknik yeterlilik, sektördeki finansal pozisyon, prosedürel uyum, iletişim vs. örnek verilebilir [7].

Tedarikçi seçimi veya değerlendirilmesi ile ilgili çalışmalar incelendiğinde 1960'lı yıllardan bu yana var olduğu görülmektedir [2]. Literatürde tedarik seçimi ile ilgili yapılan ilk çalışmalardan biri Dickson tarafından yapılmış ve bu çalışmada tedarikçi değerlendirmesinde önemli görülen 23 adet kriter belirlemiştir [7]. Dickson'ın tanımladığı bu kapsamlı kriterler günümüzde yapılan bir çok çalışmada ve işletmeler tarafindan tedarikçi seçiminde kullanılmaktadır. Yapılan çalışmalarda tedarikçi seçimi veya değerlendirilmesi konusunda en fazla kullanılan ortak kriterin kalite kriteri olduğu gözlenmiştir. Kalite kriterini sırasıyla teslimat ve maliyet kriterleri takip etmiştir. Buna ek olarak yapılan çalışmaların büyük çoğunluğunda, tedarikçinin teknik yeterliliği değerlendirme kriteri olarak kullanılmıştır. Ayrıca çalışmalar içinde aynı kriterlerin işletmenin yer aldı̆̆ sektöre göre özel tanımları ile kullanıldığı gözlenmektedir.

Belirsiz bir ortamda birbiri ile çelişen kriterlerin olması ve bu kriterlere bağlı olarak birden fazla alternatif içinden seçim yapılması nedeni ile araştırmacılar tarafından bulanık Çok Kriterli Karar Verme (ÇKKV) yöntemleri tedarikçi seçim probleminin çözümünde sıkça kullanılmaktadır. $\mathrm{Bu}$ çalışmalarda ÇKKV yöntemlerinden olan AHP, ANP, DEMATEL yöntemleri sıklıkla kullanılırken PROMETHEE I-II, TOPSIS, DEA yöntemlerinin kullanıldığı çalışmalara da rastlanmaktadır. Ayrıca belirsizlik ve az veri içeren tedarikçi seçim problemlerinde bulanık küme teorisi ve gri teori tabanlı çözüm yaklaşımlarının, hedef programlama gibi matematiksel modelleme tekniklerinin ve BWM gibi yeni yöntemlerin de tercih edildiği görülmektedir. Ayrıca otomotiv, saat, gübre, havayolu, gıda, elektronik, inşaat ve petrol\&kimya gibi birçok sektörde tedarikçi seçiminde ÇKKV yöntemleri kullanılmıştır. Ayrıca yeşil tedarikçiler ile sürdürülebilir tedarikçi seçimi konularının ele alındığı ve ÇKKV yöntemlerinin kullanılarak tedarikçi seçimi için karar destek sistemlerinin geliştirildiği çalışmalarda literatürde mevcuttur ([1], [2], [4], [6], [9], [11]- [22], [24], [26], [29], [30], [32],[33]).

$\mathrm{Bu}$ çalışmada oldukça yeni bir yöntem olan bulanık Full Consistency Method (FUCOM) yöntemi, dekoratif taş sağlayıcısı bir firmanın karar vericilerinin sübjektif değerlendirmelerine dayanarak, tedarikçi seçimi veya değerlendirilmesinde optimal kriter ağırlıklarının belirlenmesi için kullanılmıştır. Bulanık FUCOM yöntemi kriterler arasındaki tutarlılıkları dikkate alarak kriter ağırlıklarının belirlenmesini sağlamaktadır. Literatür taraması sonucunda, tedarikçi seçiminde veya değerlendirilmesinde bulanık FUCOM metodunun kullanıldığ herhangi bir çalışmaya rastlanmamıştır.

Bulanık FUCOM, Pamučar ve arkadaşları tarafından 2020'nin başlarında sunulmuştur [27]. Bu sebeple tedarikçi seçimi veya değerlendirilmesi konusunda oldukça az çalışma bulunmaktadır. Genel olarak FUCOM yönteminin bu alanda yapılan çalışmalarında inşaat firmasında sürdürülebilir tedarikçi seçiminde, kireç üretim tesisinde sürdürülebilir tedarikçi seçiminde, güneş panellerinin kurulumu için en uygun sağlayıcı firmanın seçiminde, Bosna Hersek’teki bir ahşap üretim firması için tedarikçi seçiminde, sürdürülebilir tedarik zinciri yöntemi kriterleri ile tedarikçi seçiminde kullanıldığını görülmektedir ([3], [8], [10], [23], [27], [31]).

Çalışmanın ikinci bölümünde bulanık FUCOM metodundan bahsedilmiştir. Üçüncü bölümde bu metot kullanılarak yapılan uygulama anlatılmıştır. Son bölümde sonuç ve öneriler verilmiştir.

\section{Materyal ve Metot}

\subsection{Bulanık FUCOM}

FUCOM yöntemi Pamučar et al.(2018) tarafından önerilen yeni bir ÇKKV yöntemidir. Yöntem karar vericinin önceliklerini temel alarak hem nicel hem de nitel verilerin değerlendirilebilmesi açısından ağırlıklandırma yöntemleri arasında dikkat çekmektedir. Bulanık FUCOM ilk olarak Pamučar ve arkadaşları tarafindan 2020'nin başlarında kullanılmıştır [27]. Yine 2020 yılında, çalışmasında yöntemde iyileştirmeler gerçekleştirilmiş ve nihai uygulama adımları oluşturulmuştur [26].

Bulanık FUCOM yönteminin adımları aşağıda açıklanmıştır ([26], [28], [33]).

\section{Adım 1: Karar kriterlerinin belirlenmesi}

İlk adım karar kriterlerini belirlemektir. Burada kriter sayısı j 1 'den n'e kadar $(\mathrm{j}=\{1,2,3, \ldots, \mathrm{n}\})$ olup her bir kriter değeri için $C=\left\{C_{1}, C_{2}, C_{3}, \ldots \ldots, C_{n}\right\}$ belirlenir.

\section{Adım 2: Kriterlerin Stralanması ve Üçgensel Bulanık Sayılar Kullanılarak Kriterlerin Karşılaştırılması}

Belirlenen değerlendirme kriterleri karar verici (KV) tarafından sıralanır. Sıralama, kriterlerin önemine göre yani en yüksek ağırlık katsayısına sahip olması beklenen kriterden, en düşük ağırlık katsayısına sahip olan kritere göre yapılmaktadır.

$$
C_{j(1)}>C_{j(2)},>, \ldots \ldots, C_{j(k)}
$$

Burada $C_{j(k)}, \mathrm{j}=\{1,2, \ldots . \mathrm{n}\} \quad$ kriteri gösterirken $\mathrm{k}, C_{j}$ kriterlerinin karar verici için sırasını temsil eder. Eğer arada eşitlik varsa "=" işareti konur.

Üçgensel bulanık sayı operatörleri ile işlem yapılmıştır [5]. Sıralanan kriterlerin bir karşılaştırması yapılır ve karşılaştırmalı öncelik vektörleri $\varphi_{(k / k+1)}$ oluşturulur. Burada $\varphi_{(k / k+1)} \mathrm{k}$. 
sıralamaya sahip kriterin $(\mathrm{k}+1)$. siralamaya sahip kritere olan üstünlük değerini ifade eder.

$$
\begin{gathered}
\Phi=\left(\varphi_{(1 / 2)}, \varphi_{(2 / 3)}, \ldots, \varphi_{(k /(k+1)}\right) \\
\varphi_{(k /(k+1))}=\frac{\varpi_{C_{j(k)}}}{\varpi_{C_{j(k+1)}}} \\
=\frac{\varpi_{C_{j(k+1)}^{l},}, \varpi_{C_{j(k+1)}^{m}}, \varpi_{C_{j(k+1)}^{u}}}{\varpi_{C_{j(k)}^{l}}^{l}, \varpi_{C_{j(k)}^{m}}, \varpi_{C_{j(k)}^{u}}^{u}}
\end{gathered}
$$

Burada, $\varpi_{C_{j(k)}}$ k.sıralamadaki j. olarak belirlenen kriterin önem derecesini göstermektedir.

\section{Adım 3: Optimum bulanık ăğırlıkların hesaplanması.}

Bu son adımda kriterlerin nihai ağırlığı elde edilir. Bu ağırlık değerleri elde edilirken aşağıdaki iki koşulu sağlamalıdır:

Kriterlerin önem katsayılarının oranının $\left(\frac{w_{k}}{w_{(k+1)}}\right)$, Adım 2'de kriterler için tanımlanan karşılaştırmalı öncelik vektörüne $\left(\varphi_{k /(k+1)}\right)$ eşit olması; yani, aşağıdaki Eşitlik(4)'ü sağlaması gerekmektedir.

$$
\frac{w_{k}}{w_{(k+1)}}=\varphi_{k /(k+1)}
$$

Eşitlik(4)'e ek olarak, ağırlık katsayılarının nihai değerleri matematiksel geçişlilik koşulunu karşılamalıdır. Eşitlik(5) ve Eşitlik(6) yardımıyla, gerekli olan bir diğer koşul, Eşitlik(7) oluşturulur.

$$
\begin{gathered}
\frac{w_{k}}{w_{(k+2)}}=\frac{w_{k}}{w_{(k+1)}} \otimes \frac{w_{(k+1)}}{w_{(k+2)}} \\
\frac{\varphi_{k}}{\varphi_{(k+2)}}=\frac{\varphi_{k}}{\varphi_{(k+1)}} \otimes \frac{\varphi_{(k+1)}}{\varphi_{(k+2)}} \\
\frac{w_{k}}{w_{(k+2)}}=\varphi_{k /(k+1)} \otimes \varphi_{(k+1) /(k+2)}
\end{gathered}
$$

Yukarıda belirtilen Eşitlik(4) ve Eşitlik(5) sağlandığında maksimum tutarlılık gerçekleşir. Bu durumda DMC (Deviation from maximum Consistency) denilen tam tutarlılıktan sapma katsayısının değeri minimum olur. Eşitlik(8)'de verilen matematiksel model çözülerek nihai ağırlıkların $\left(w_{1}, w_{2}, w_{3}, \ldots, w_{n}\right)$ son değerleri ve DMC değeri, $\chi$ elde edilerek kriter ağırlıkları tahsis edilir. $\min \chi$

s.t.

$$
\begin{aligned}
& \left\{\begin{array}{c}
\left|w_{j(k)}-w_{(j)(k+1)} \otimes \frac{\varphi_{k}}{\varphi_{(k+1)}}\right| \leq \chi, \forall_{j} \\
\left|w_{j(k)}-w_{(j)(k+2)} \otimes \frac{\varphi_{k}}{\varphi_{(k+1)}} \otimes \frac{\varphi_{(k+1)}}{\varphi_{(k+2)}}\right| \leq \chi, \forall_{j} \\
\sum_{j=1}^{n} w_{j}=1, \forall_{j}
\end{array}\right. \\
& w_{j}^{l} \leq w_{j}^{m} \leq w_{j}^{u} \\
& w_{j}^{l} \geq 0, \forall_{j} \\
& j=1,2,3, \ldots, n \\
& w_{j}=\left(w_{j}^{l}, w_{j}^{m}, w_{j}^{u}\right), \\
& \varphi_{k /(k+1)}=\left(\varphi_{k /(k+1)}^{l}, \varphi_{k /(k+1)}^{m}, \varphi_{k /(k+1)}^{u}\right) \quad \text { ve } \quad \varphi_{\frac{(k+1)}{(k+2)}}=
\end{aligned}
$$

\section{Araştırma Sonuçları ve Tartışma}

Uygulama kapsamında Boya\&Kimya sektöründe bir firmanın dekoratif taş tedarikçileri değerlendirilmesinde kullanılacak kriterlerin ağırlıkları belirlenmiştir [33].

\section{Adım 1: Karar kriterlerinin belirlenmesi}

İlk olarak, kriter belirleyecek ve kriter önem ağırlarını değerlendirecek KV'ler belirlenmiştir. Uygulamanın yapıldığ firmada satın alma, pazarlama ve kalite departmanlarında görev yapan ve 8 ile 15 yıl arasında tecrübeye sahip 4 personel KV'ler olarak seçilmiştir.

Tedarikçi seçim kriterlerin belirlenmesi için ilk olarak literatür taraması yapılmış ve belirlenen kriterler firmanın sahip olduğu çalışma koşulları ve yönetim politikalar dikkate alınarak tanımlanmıştır [7]. Daha sonra KV'lerin tarafından bu kriterler onaylanmıştır. Tablo 1'de kriterler tanımlamaları ile birlikte verilmiştir.

Tablo 1. Kriter Değerlendirme Tablosu

\begin{tabular}{l|l}
\hline Kriterler & Kriter Açıklamaları \\
\hline Tasarım $\left(\mathrm{C}_{1}\right)$ & Taşın renk, boyut ve şekil uygunluğu \\
\hline Maliyet $\left(\mathrm{C}_{2}\right)$ & $\begin{array}{l}\text { Ürünün birim maliyeti ve firmanın ödeme } \\
\text { vadesi uygunluğu }\end{array}$ \\
\hline $\begin{array}{l}\text { Uygulama } \\
\text { Kolaylığı }\left(\mathrm{C}_{3}\right)\end{array}$ & Gönderilen taşın kullanılabilirliği \\
\hline Kalite $\left(\mathrm{C}_{4}\right)$ & İstenilen kalitede olması \\
\hline $\begin{array}{l}\text { Stok } \\
\text { Durumu }\left(\mathrm{C}_{5}\right)\end{array}$ & Sipariş miktarını karşılama kapasitesi \\
\hline $\begin{array}{l}\text { Nakliye } \\
\text { Durumu }\left(\mathrm{C}_{6}\right)\end{array}$ & Zamanında sevkiyat yapabilme yeterliliği \\
\hline $\begin{array}{l}\text { Geçmiş } \\
\text { Performans }\left(\mathrm{C}_{7}\right)\end{array}$ & $\begin{array}{l}\text { Geçmiş işler için tedarikçinin kalite, } \\
\text { maliyet ve nakliye uyumu }\end{array}$ \\
\hline $\begin{array}{l}\text { Garanti\& } \\
\text { Politikalar }\left(\mathrm{C}_{8}\right)\end{array}$ & $\begin{array}{l}\text { Şirket politika ve standartlarına uyarak } \\
\text { ürünü eleçleme, nakliye ve tedarik } \\
\text { zamanına uyum. Hasarlı ürün tespitinde } \\
\text { verilen tepki ve iyileştirme çabaları }\end{array}$ \\
\hline $\begin{array}{l}\text { Teknik } \\
\text { Yeterlilik }\left(\mathrm{C}_{9}\right)\end{array}$ & $\begin{array}{l}\text { Özelleştirilmiş olarak istenilen ürünü } \\
\text { üretme yeteneği }\end{array}$ \\
\hline
\end{tabular}




\begin{tabular}{|c|c|}
\hline $\begin{array}{l}\text { İletişim } \\
\text { Sistemi }\left(\mathrm{C}_{10}\right)\end{array}$ & $\begin{array}{l}\text { Tedarikçiye istenildiğinde ulaşma ve } \\
\text { tedarik süreçlerinin takibinin sağlanması }\end{array}$ \\
\hline $\begin{array}{l}\text { İtibar ve } \\
\text { Endüstrideki } \\
\text { Durum }\left(\mathrm{C}_{11}\right)\end{array}$ & Tedarikçinin sektördeki konumu \\
\hline $\begin{array}{l}\text { Eğitim } \\
\text { Destekleri }\left(\mathrm{C}_{12}\right)\end{array}$ & $\begin{array}{l}\text { Ürünün kullanım alanı ve kullanım } \\
\text { biçimine ait firmaya verdiği bilgi ve } \\
\text { doküman yeterliliği }\end{array}$ \\
\hline
\end{tabular}

Adım 2: Kriterlerin Stralanması ve Üçensel Bulanık Sayılar Kullanılarak Kriterlerin Karşılaştırılması

Herbir karar verici tarafından ilk olarak kriterler önem sırasına göre sıralanmıştır. Kriter önceliklendirilmesi için Tablo 2'de gösterilen ölçek kullanılmıştır [5].

Tablo 2. Kriter değerlendirme için dilsel ifadelerin üyelik fonksiyon değerleri

\begin{tabular}{l|l}
\hline Dilsel İfadeler & Bulanık Sayı Değerleri \\
\hline Eşit Derecede Önemli $(\mathrm{OZ})$ & $(1,1,1)$ \\
\hline Çok Zayıf Önemli(ÇZ) & $(1,2,3)$ \\
\hline Zayıf Önemli(Z) & $(2,3,4)$ \\
\hline Orta Zayıf Önemli(RZ) & $(3,4,5)$ \\
\hline Orta Önemli (O) & $(4,5,6)$ \\
\hline Daha Önemli (RI) & $(5,6,7)$ \\
\hline Oldukça Önemli (İ) & $(6,7,8)$ \\
\hline Çok Önemli (ÇI) & $(7,8,9)$ \\
\hline Mükemmel önemli (Oİ) & $(8,9,10)$ \\
\hline
\end{tabular}

Sıralama işlemi, en yüksek önem katsayısına sahip olması beklenen kriterden, en düşük önem katsayısına sahip olan kritere göre Eşitlik (1) yardımıyla yapılmıştır:

$$
\begin{aligned}
& K V_{1}: \mathrm{C}_{2}=\mathrm{C}_{4}=\mathrm{C}_{1}>\mathrm{C}_{5}=\mathrm{C}_{6}=\mathrm{C}_{3}>\mathrm{C}_{11}=\mathrm{C}_{12}>\mathrm{C}_{7}=\mathrm{C}_{8} \\
& >\mathrm{C}_{10}=\mathrm{C}_{9} \\
& K V_{2}: \mathrm{C}_{4}=\mathrm{C}_{5}=\mathrm{C}_{6}>\mathrm{C}_{1}>\mathrm{C}_{10}>\mathrm{C}_{12}=\mathrm{C}_{9}=\mathrm{C}_{11}>\mathrm{C}_{7}=\mathrm{C}_{2} \\
& >\mathrm{C}_{8}=\mathrm{C}_{3}
\end{aligned}
$$

$$
\begin{aligned}
& K V_{3}: \mathrm{C}_{5}=\mathrm{C}_{6}=\mathrm{C}_{9}>\mathrm{C}_{12}>\mathrm{C}_{2}=\mathrm{C}_{1}=\mathrm{C}_{4}=\mathrm{C}_{7}>\mathrm{C}_{8}=\mathrm{C}_{3} \\
& >\mathrm{C}_{11}>\mathrm{C}_{10} \\
& K V_{4}: \mathrm{C}_{1}=\mathrm{C}_{4}>\mathrm{C}_{2}=\mathrm{C}_{5}=\mathrm{C}_{6}>\mathrm{C}_{7}>\mathrm{C}_{3}=\mathrm{C}_{8}=\mathrm{C}_{9}>\mathrm{C}_{12} \\
& >\mathrm{C}_{10}>\mathrm{C}_{11}
\end{aligned}
$$

Karar vericiler Tablo 2'de gösterilen bulanık değerlendirme ölçeğini kullanarak önem sıralamasında ilk sırada belirledikleri kriterin bir sonraki kriterlere göre önemlilik düzeylerini belirlemişlerdir. Dilsel değerlendirmeler bulanık sayı değerlerine çevrilerek Tablo 3 'de sunulmuştur.

Tablo 3. KV'lerin bulanık sayı değerleri ile değerlendirmeleri

\begin{tabular}{l|l|l|l|l}
\hline & $\mathbf{K V}_{\mathbf{1}}$ & $\mathbf{K} \mathbf{V}_{\mathbf{2}}$ & $\mathbf{K V}$ & $\mathbf{K} \mathbf{V}_{\mathbf{4}}$ \\
\hline $\mathbf{C}_{\mathbf{1}}$ & $(1,1,1)$ & $(1,2,3)$ & $(2,3,4)$ & $(1,1,1)$ \\
\hline $\mathbf{C}_{\mathbf{2}}$ & $(1,1,1)$ & $(4,5,6)$ & $(2,3,4)$ & $(1,2,3)$ \\
\hline $\mathbf{C}_{\mathbf{3}}$ & $(1,2,3)$ & $(5,6,7)$ & $(3,4,5)$ & $(3,4,5)$ \\
\hline $\mathbf{C}_{\mathbf{4}}$ & $(1,1,1)$ & $(1,1,1)$ & $(2,3,4)$ & $(1,1,1)$ \\
\hline $\mathbf{C}_{\mathbf{5}}$ & $(1,2,3)$ & $(1,1,1)$ & $(1,1,1)$ & $(1,2,3)$ \\
\hline $\mathbf{C}_{\mathbf{6}}$ & $(1,2,3)$ & $(1,1,1)$ & $(1,1,1)$ & $(1,2,3)$ \\
\hline $\mathbf{C}_{\mathbf{7}}$ & $(3,4,5)$ & $(4,5,6)$ & $(2,3,4)$ & $(2,3,4)$ \\
\hline $\mathbf{C}_{\mathbf{8}}$ & $(3,4,5)$ & $(5,6,7)$ & $(3,4,5)$ & $(3,4,5)$ \\
\hline $\mathbf{C}_{\mathbf{9}}$ & $(4,5,6)$ & $(3,4,5)$ & $(1,1,1)$ & $(3,4,5)$ \\
\hline $\mathbf{C}_{\mathbf{1 0}}$ & $(4,5,6)$ & $(2,3,4)$ & $(5,6,7)$ & $(5,6,7)$ \\
\hline $\mathbf{C}_{\mathbf{1 1}}$ & $(2,3,4)$ & $(3,4,5)$ & $(4,5,6)$ & $(7,8,9)$ \\
\hline $\mathbf{C}_{\mathbf{1 2}}$ & $(2,3,4)$ & $(3,4,5)$ & $(1,2,3)$ & $(4,5,6)$ \\
\hline
\end{tabular}

\section{Adım 3. Optimum Bulanık Ăğırlıkların Hesaplanması}

Kriterlerin karşılaştırmalı önemi Eşitlik (2) ve Eşitlik (3) yardımıyla, her bir KV için Tablo 4'deki gibi tanımlanmıştır.

\begin{tabular}{|c|c|c|c|c|c|c|c|}
\hline \multicolumn{2}{|c|}{$\mathbf{K} \mathbf{V}_{1}$} & \multicolumn{2}{|c|}{$K_{2}$} & \multicolumn{2}{|c|}{$\mathrm{KV}_{\mathbf{3}}$} & \multicolumn{2}{|c|}{$\mathbf{K} \mathbf{V}_{4}$} \\
\hline$C_{2}$ & - & $\mathrm{C}_{4}$ & - & $\mathrm{C}_{5}$ & - & $C_{1}$ & - \\
\hline $\mathrm{C}_{4}$ & $(1,1,1)$ & $C_{5}$ & $(1,1,1)$ & $\mathrm{C}_{6}$ & $(1,1,1)$ & $\mathrm{C}_{4}$ & $(1,1,1)$ \\
\hline$C_{1}$ & $(1,1,1)$ & $C_{6}$ & $(1,1,1)$ & $C_{9}$ & $(1,1,1)$ & $\mathrm{C}_{2}$ & $(1,2,3)$ \\
\hline $\mathrm{C}_{5}$ & $(1,2,3)$ & $C_{1}$ & $(1,2,3)$ & $C_{12}$ & $(1,2,3)$ & $\mathrm{C}_{5}$ & $(0.333,1,3)$ \\
\hline $\mathrm{C}_{6}$ & $(0.333,1,3)$ & $\mathrm{C}_{10}$ & $(0.667,1.5,4)$ & $\mathrm{C}_{2}$ & $(0.667,1.5,4)$ & $\mathrm{C}_{6}$ & $(0.333,1,3)$ \\
\hline $\mathrm{C}_{3}$ & $(0.333,1,3)$ & $\mathrm{C}_{12}$ & $(0.75,1.333,2.5)$ & $C_{1}$ & $(0.5,1,2)$ & $\mathbf{C}_{7}$ & $(0.667,1.5,4)$ \\
\hline $\mathrm{C}_{11}$ & $(0.667,1.5,4)$ & $\mathrm{C}_{9}$ & $(0.6,1,1.667)$ & $\mathrm{C}_{4}$ & $(0.5,1,2)$ & $\mathrm{C}_{3}$ & $(0.75,1.333,2.5)$ \\
\hline$C_{12}$ & $(0.5,1,2)$ & $\mathrm{C}_{11}$ & $(0.6,1,1.667)$ & $C_{7}$ & $(0.5,1,2)$ & $\mathrm{C}_{8}$ & $(0.6,1,1.667)$ \\
\hline $\mathrm{C}_{7}$ & $(0.75,1.333,2.5)$ & $C_{7}$ & $(0.8,1.25,2)$ & $\mathrm{C}_{8}$ & $(0.75,1.333,2.5)$ & $\mathrm{C}_{9}$ & $(0.6,1,1.667)$ \\
\hline $\mathrm{C}_{8}$ & $(0.6,1,1.667)$ & $\mathrm{C}_{2}$ & $(0.667,1,1.5)$ & $C_{3}$ & $(0.6,1,1.667)$ & $C_{12}$ & $(0.8,1.25,2)$ \\
\hline $\mathrm{C}_{10}$ & $(0.8,1.25,2)$ & $\mathrm{C}_{8}$ & $(0.833,1.2,1.75)$ & $\mathrm{C}_{11}$ & $(0.8,1.25,2)$ & $C_{10}$ & $(0.833,1.2,1.75)$ \\
\hline $\mathrm{C}_{9}$ & $(0.667,1,1.5)$ & $C_{3}$ & $(0.714,1,1.4)$ & $\mathbf{C}_{10}$ & $(0.833,1.2,1.75)$ & $\mathbf{C}_{11}$ & $(1,1.333,1.8)$ \\
\hline
\end{tabular}
Karşılaştırmalı öncelik vektörlerine ek olarak, Eşitlik(7) ile belirtilen matematiksel geçerlilik koşulu sağlanmalıdır. $\mathrm{Bu}$ sebeple $\varphi_{(k / k+2)}$ 'nin değeri hesaplanmalıdır. Bu aşamada (n-2) adet yani 10 adet karşılaştırma bulunmaktadır. Her bir KV için $\varphi_{(k / k+2)}$ karşılaştırmalı öncelik değerleri sırasıyla Tablo 5'te gösterildiği gibi hesaplanmıştır.

Tablo 4. KV'ler için $\varphi_{(k / k+1)}$ karşılaştırmalı öncelik değerleri 
European Journal of Science and Technology

Tablo 5. KV'ler için $\varphi_{(k / k+2)}$ karşılaştırmalı öncelik değerleri

\begin{tabular}{|c|c|c|c|c|c|c|c|}
\hline \multicolumn{2}{|l|}{$\mathbf{K} \mathbf{V}_{1}$} & \multicolumn{2}{|l|}{$K_{V_{2}}$} & \multicolumn{2}{|c|}{$\mathrm{KV}_{3}$} & \multicolumn{2}{|c|}{$\mathrm{KV}_{4}$} \\
\hline $\mathbf{C}_{2}$ & - & $\mathrm{C}_{4}$ & - & $\mathrm{C}_{5}$ & - & $\mathbf{C}_{1}$ & - \\
\hline $\mathrm{C}_{4}$ & - & $\mathrm{C}_{5}$ & - & $C_{6}$ & - & $\mathrm{C}_{4}$ & - \\
\hline$C_{1}$ & $(1,1,1)$ & $C_{6}$ & $(1,1,1)$ & C9 & $(1,1,1)$ & $\mathrm{C}_{2}$ & $(1,2,3)$ \\
\hline $\mathrm{C}_{5}$ & $(1,2,3)$ & $\mathbf{C}_{1}$ & $(1,2,3)$ & $\mathrm{C}_{12}$ & $(1,2,3)$ & $\mathrm{C}_{5}$ & $(0.333,2,9)$ \\
\hline $\mathrm{C}_{6}$ & $(0.333,2,9)$ & $\mathrm{C}_{10}$ & $(0.667,3,12)$ & $\mathrm{C}_{2}$ & $(0.667,3,12)$ & $\mathrm{C}_{6}$ & $(0.111,1,9)$ \\
\hline $\mathbf{C}_{3}$ & $(0.111,1,9)$ & $\mathrm{C}_{12}$ & $(0.5,2,10)$ & $\mathrm{C}_{1}$ & $(0.333,1.5,8)$ & $\mathbf{C}_{7}$ & $(0.222,1.5,12)$ \\
\hline $\mathrm{C}_{11}$ & $(0.222,1.5,12)$ & $\mathrm{C}_{9}$ & $(0.45,1.333,4.167)$ & $\mathrm{C}_{4}$ & $(0.25,1,4)$ & $\mathrm{C}_{3}$ & $(0.5,2,10)$ \\
\hline $\mathrm{C}_{12}$ & $(0.333,1.5,8)$ & $\mathrm{C}_{11}$ & $(0.36,1,2.778)$ & $\mathbf{C}_{7}$ & $(0.25,1,4)$ & $\mathrm{C}_{8}$ & $(0.45,1.333,4.167)$ \\
\hline $\mathbf{C}_{7}$ & $(0.375,1.333,5)$ & $\mathbf{C}_{7}$ & $(0.48,1.25,3.333)$ & $\mathrm{C}_{8}$ & $(0.375,1.333,5)$ & $\mathrm{C}_{9}$ & $(0.36,1,2.778)$ \\
\hline $\mathrm{C}_{8}$ & $(0.45,1.333,4.167)$ & $\mathrm{C}_{2}$ & $(0.533,1.25,3)$ & $\mathrm{C}_{3}$ & $(0.45,1.333,4.167)$ & $\mathrm{C}_{12}$ & $(0.48,1.25,3.333)$ \\
\hline $\mathrm{C}_{10}$ & $(0.48,1.25,3.333)$ & $\mathrm{C}_{8}$ & $(0.556,1.2,2.625)$ & $\mathrm{C}_{11}$ & $(0.48,1.25,3.333)$ & $\mathbf{C}_{10}$ & $(0.667,1.5,3.5)$ \\
\hline $\mathrm{C}_{9}$ & $(0.533,1.25,3)$ & $\mathrm{C}_{3}$ & $(0.595,1.2,2.45)$ & $\mathrm{C}_{10}$ & $(0.667,1.5,3.5)$ & $\mathrm{C}_{11}$ & $(0.833,1.6,3.15)$ \\
\hline
\end{tabular}

Her bir KV için matematiksel model Eşitlik (8) ile oluşturulmuş ve LİNGO 18.0 kullanılarak çözülmüştür. Örnek olarak; $K V_{1}$ için oluşturulan matematiksel model aşağıda verilmiştir [33].

$\min \chi$

st.

$$
\begin{gathered}
\left|w_{2}^{l}-1 \cdot w_{4}^{u}\right| \leq \chi ;\left|w_{2}^{m}-1 \cdot w_{4}^{m}\right| \leq \chi ; \\
\left|w_{2}^{u}-1 \cdot w_{4}^{l}\right| \leq \chi ;\left|w_{4}^{l}-1 \cdot w_{1}^{u}\right| \leq \chi ; \\
\left|w_{2}^{l}-1 \cdot w_{4}^{u}\right| \leq \chi ;\left|w_{2}^{m}-1 \cdot w_{4}^{m}\right| \leq \chi ; \\
\left|w_{2}^{u}-1 \cdot w_{4}^{l}\right| \leq \chi ;\left|w_{4}^{l}-1 \cdot w_{1}^{u}\right| \leq \chi ; \\
\left|w_{4}^{m}-1 \cdot w_{1}^{m}\right| \leq \chi ;\left|w_{4}^{u}-1 \cdot w_{1}^{l}\right| \leq \chi ; \\
\left|w_{1}^{l}-1 \cdot w_{5}^{u}\right| \leq \chi ;\left|w_{1}^{m}-2 \cdot w_{5}^{m}\right| \leq \chi ; \\
\left|w_{1}^{u}-3 \cdot w_{5}^{l}\right| \leq \chi ;\left|w_{5}^{l}-0.333 \cdot w_{6}^{u}\right| \leq \chi ; \\
\left|w_{5}^{m}-1 \cdot w_{6}^{m}\right| \leq \chi ;\left|w_{5}^{u}-3 \cdot w_{6}^{l}\right| \leq \chi ; \\
\left|w_{6}^{l}-0.333 \cdot w_{3}^{u}\right| \leq \chi ;\left|w_{6}^{m}-1 \cdot w_{3}^{m}\right| \leq \chi ; \\
\left|w_{6}^{u}-3 \cdot w_{3}^{l}\right| \leq \chi ;\left|w_{3}^{l}-0.667 \cdot w_{11}^{u}\right| \leq \chi ; \\
\left|w_{3}^{m}-1.5 \cdot w_{11}^{m}\right| \leq \chi ;\left|w_{3}^{u}-4 \cdot w_{11}^{l}\right| \leq \chi ; \\
\left|w_{11}^{l}-0.5 \cdot w_{12}^{u}\right| \leq \chi ;\left|w_{11}^{m}-1 \cdot w_{12}^{m}\right| \leq \chi ; \\
\left|w_{11}^{u}-2 \cdot w_{12}^{l}\right| \leq \chi ;\left|w_{12}^{l}-0.75 \cdot w_{7}^{u}\right| \leq \chi ; \\
\left|w_{12}^{m}-1.333 \cdot w_{7}^{m}\right| \leq \chi ;\left|w_{12}^{u}-2.5 \cdot w_{7}^{l}\right| \leq \chi ; \\
\left|w_{7}^{l}-0.6 \cdot w_{8}^{u}\right| \leq \chi ;\left|w_{7}^{m}-1 \cdot w_{8}^{m}\right| \leq \chi ; \\
\left|w_{7}^{u}-1.667 \cdot w_{8}^{l}\right| \leq \chi ;\left|w_{8}^{l}-0.8 \cdot w_{10}^{u}\right| \leq \chi ; \\
\left|w_{8}^{m}-1.25 \cdot w_{10}^{m}\right| \leq \chi ;\left|w_{8}^{u}-2 \cdot w_{10}^{l}\right| \leq \chi ; \\
\left|w_{8}^{l}-0.8 \cdot w_{10}^{u}\right| \leq \chi ;\left|w_{8}^{m}-1.25 \cdot w_{10}^{m}\right| \leq \chi ; \\
\left|w_{8}^{u}-2 \cdot w_{10}^{l}\right| \leq \chi ;
\end{gathered}
$$$$
\left|w_{2}^{l}-1 \cdot w_{1}^{u}\right| \leq \chi ;\left|w_{2}^{m}-1 \cdot w_{1}^{m}\right| \leq \chi ;
$$$$
\left|w_{2}^{u}-1 \cdot w_{1}^{l}\right| \leq \chi ;\left|w_{4}^{l}-1 \cdot w_{5}^{u}\right| \leq \chi ;
$$$$
\left|w_{4}^{m}-2 \cdot w_{5}^{m}\right| \leq \chi ;\left|w_{4}^{u}-3 \cdot w_{5}^{l}\right| \leq \chi ;
$$$$
\left|w_{1}^{l}-0.333 \cdot w_{6}^{u}\right| \leq \chi ;\left|w_{1}^{m}-2 \cdot w_{6}^{m}\right| \leq \chi ;
$$$$
\left|w_{1}^{u}-9 \cdot w_{6}^{l}\right| \leq \chi ;\left|w_{5}^{l}-0.111 \cdot w_{3}^{u}\right| \leq \chi ;
$$$$
\left|w_{5}^{m}-1 \cdot w_{3}^{m}\right| \leq \chi ;\left|w_{5}^{u}-9 \cdot w_{3}^{l}\right| \leq \chi ;
$$$$
\left|w_{6}^{l}-0.222 \cdot w_{11}^{u}\right| \leq \chi ;\left|w_{6}^{m}-1.5 \cdot w_{11}^{m}\right| \leq \chi ;
$$$$
\left|w_{6}^{u}-12 \cdot w_{11}^{l}\right| \leq \chi ;\left|w_{3}^{l}-0.333 \cdot w_{12}^{u}\right| \leq \chi ;
$$$$
\left|w_{3}^{m}-1.5 \cdot w_{12}^{m}\right| \leq \chi ;\left|w_{3}^{u}-8 \cdot w_{12}^{l}\right| \leq \chi ;
$$$$
\left|w_{11}^{l}-0.375 \cdot w_{7}^{u}\right| \leq \chi ;\left|w_{11}^{m}-1.333 \cdot w_{7}^{m}\right| \leq \chi ;
$$$$
\left|w_{11}^{u}-5 \cdot w_{7}^{l}\right| \leq \chi ;\left|w_{12}^{l}-0.45 \cdot w_{8}^{u}\right| \leq \chi ;
$$

$$
\begin{gathered}
\left|w_{12}^{m}-1.333 \cdot w_{8}^{m}\right| \leq \chi ;\left|w_{12}^{u}-4.167 \cdot w_{8}^{l}\right| \leq \chi ; \\
\left|w_{7}^{l}-0.48 \cdot w_{10}^{u}\right| \leq \chi ;\left|w_{7}^{m}-1.25 \cdot w_{10}^{m}\right| \leq \chi \\
\left|w_{7}^{u}-3.333 \cdot w_{10}^{l}\right| \leq \chi ;\left|w_{8}^{l}-0.53 \cdot w_{9}^{u}\right|, \leq \chi \\
\left|w_{8}^{m}-1.25 \cdot w_{9}^{m}\right| \leq \chi ;\left|w_{8}^{u}-3 \cdot w_{9}^{l}\right| \leq \chi
\end{gathered}
$$

$$
\begin{gathered}
1 / 6\left(w_{1}^{l}+4 \cdot w_{1}^{m}+w_{1}^{u}\right)+1 / 6\left(w_{2}^{l}+4 \cdot w_{2}^{m}+w_{2}^{u}\right) \\
+1 / 6\left(w_{3}^{l}+4 \cdot w_{3}^{m}+w_{3}^{u}\right)+1 / 6\left(w_{1}^{l}+4 \cdot w_{1}^{m}+w_{1}^{u}\right) \\
+1 / 6\left(w_{2}^{l}+4 \cdot w_{2}^{m}+w_{2}^{u}\right)+1 / 6\left(w_{3}^{l}+4 \cdot w_{3}^{m}+w_{3}^{u}\right) \\
+1 / 6\left(w_{4}^{l}+4 \cdot w_{4}^{m}+w_{4}^{u}\right)+1 / 6\left(w_{5}^{l}+4 \cdot w_{5}^{m}+w_{5}^{u}\right) \\
+1 / 6\left(w_{6}^{l}+4 \cdot w_{6}^{m}+w_{6}^{u}\right)+1 / 6\left(w_{7}^{l}+4 \cdot w_{7}^{m}+w_{7}^{u}\right) \\
+1 / 6\left(w_{8}^{l}+4 \cdot w_{8}^{m}+w_{8}^{u}\right)+1 / 6\left(w_{9}^{l}+4 \cdot w_{9}^{m}+w_{9}^{u}\right) \\
+1 / 6\left(w_{10}^{l}+4 \cdot w_{10}^{m}+w_{10}^{u}\right)+1 / 6\left(w_{11}^{l}+4 \cdot w_{11}^{m}+w_{11}^{u}\right) \\
+1 / 6\left(w_{12}^{l}+4 \cdot w_{12}^{m}+w_{12}^{u}\right)=1 ; \\
w_{1}^{l} \leq w_{1}^{m} \leq w_{1}^{u}, w_{2}^{l} \leq w_{2}^{m} \leq w_{2}^{u}, w_{3}^{l} \leq w_{3}^{m} \leq w_{3}^{u}, \\
w_{4}^{l} \leq w_{4}^{m} \leq w_{4}^{u}, w_{5}^{l} \leq w_{5}^{m} \leq w_{5}^{u}, w_{6}^{l} \leq w_{6}^{m} \leq w_{6}^{u}, \\
w_{7}^{l} \leq w_{7}^{m} \leq w_{7}^{u}, w_{8}^{l} \leq w_{8}^{m} \leq w_{8}^{u}, w_{9}^{l} \leq w_{9}^{m} \leq w_{9}^{u}, \\
w_{10}^{l} \leq w_{10}^{m} \leq w_{10}^{u}, w_{11}^{l} \leq w_{11}^{m} \leq w_{11}^{u}, w_{12}^{l} \leq w_{12}^{m} \leq w_{12}^{u} ; \\
w_{1}^{l}, w_{2}^{l}, w_{3}^{l}, w_{4}^{l}, w_{5}^{l}, w_{6}^{l}, w_{7}^{l}, w_{8}^{l}, w_{9}^{l}, w_{10}^{l}, w_{11}^{l}, w_{12}^{l} \geq 0 .
\end{gathered}
$$

Matematiksel modelin çözümü sonucunda, $\chi_{D M_{1}}=$ $0.039, \chi_{D M_{2}}=0.043, \chi_{D M_{3}}=0.033, \chi_{D M_{4}}=0.0380$ olarak bulunmuştur. Hesaplanan bulanık kriter ağırlıkları Eşitlik (21) kullanılarak durusallaştırılmıştır. Her bir KV için ayrı ayrı elde edilen kriterler ağırlıkları, nihai kriter ağırlık değerlerini bulmak için geometrik ortalamaya başvurulmuştur ve sonrasında bu ağırlıklar normalleştirilmiştir. Kriterler için bulunan nihai ağırlık değerleri: $\mathrm{C}_{1}=0.079, \mathrm{C}_{2}=0.082, \mathrm{C}_{3}=0.08, \mathrm{C}_{4}=0.113, \mathrm{C}_{5}=0.096$, $\mathrm{C}_{6}=0.085, \mathrm{C}_{7}=0.076, \mathrm{C}_{8}=0.084, \mathrm{C}_{9}=0.094, \mathrm{C}_{10}=0.069, \mathrm{C}_{11}=0.074$, $\mathrm{C}_{12}=0.067$ olarak hesaplanmıştır. $\mathrm{Bu}$ ağırlıklara göre tedarikçi değerlendirmesinde en önemli kriter kalite kriteri ve en önemsiz kriter ise eğitim destekleri kriteri olarak belirlenmiştir. Firma bu kriter ağılıklarını dikkate alarak tedarikçilerini değerlendirmelidir. 


\section{Sonuç ve Öneriler}

$\mathrm{Bu}$ çalışmada tedarik zinciri kavramının paydaşlarından biri olan tedarikçi kavramı üzerine durulmuş; bir firmanın tedarikçileri değerlendirilmiştir. KV'lerin sübjektif değerlendirmeleri kullanılanan ve doğrusal olmayan programlama modeline dayanan bulanık FUCOM metodu ile DMC değerini bağlı olarak optimum kriter ağırlıkları belirlenmiştir.

Gelecekte yapılacak çalışmalarda tedarikçi seçimde sürdürülebilirlik kriterleri dikkate alınabilir. Farklı sektörlerde ve uygulanan sektöre göre seçim kriterlerinin özelleştirilmesi ile çalışma farklı bir boyut kazanacaktır. Ayrıca farklı ÇKKV yöntemleri kullanılarak tedarikçi alternatifi seçimi yapılabilir.

\section{Teșekkür}

Karar vericilere ve uygulamanın yapıldığı işletme yetkililerine teşekkür ederiz.

\section{Kaynakça}

[1] Abdollahi, M., Arvan, M., \& Razmi, J., 2015. An integrated approach for supplier portfolio selection: lean or agile. Expert Systems with Applications, 42(1), 679-690. doi:10.1016/j.eswa.2014.08.019

[2] Alavi, B., Tavana, M., \& Mina, H., 2021. A dynamic decision support system for sustainable supplier selection in circular economy. Sustainable Production and Consumption, 27, 905-920. doi:10.1016/j.spc.2021.02.015

[3] Cao, Esangbedo, Bai, \& Esangbedo., 2019. Grey SWARA-FUCOM weighting method for contractor selection mcdm problem: a case study of floating solar panel energy system installation. Energies, 12(13). doi:10.3390/en12132481

[4] Cengiz, A. E., Aytekin, O., Ozdemir, I., Kusan, H., \& Cabuk, A., 2017. A multi-criteria decision model for construction material supplier selection. Procedia Engineering, 196, 294-301. doi:10.1016/j.proeng.2017.07.202

[5] Chou, Y.-C., Sun, C.-C., \& Yen, H.-Y., 2012. Evaluating the criteria for human resource for science and technology (HRST) based on an integrated fuzzy AHP and fuzzy DEMATEL approach. Applied Soft Computing, 12(1), 64-71. doi:10.1016/j.asoc.2011.08.058

[6] Dargi, A., Anjomshoae, A., Galankashi, M. R., Memari, A., \& Tap, M. B. M., 2014. Supplier selection: a Fuzzy-ANP approach. Procedia Computer Science, 31, 691-700. doi:10.1016/j.procs.2014.05.317

[7] Dickson, G. W. (1966). An analysis of vendor selection systems and decisions. Journal of purchasing, 2(1), 5-17.

[8] Durmić, E., Stević, Ž., Chatterjee, P., Vasiljević, M., \& Tomašević, M., 2020. Sustainable supplier selection using combined FUCOM Rough SAW model. Reports in Mechanical Engineering, 1(1), 34-43. doi:10.31181/rme200101034c

[9] Dweiri, F., Kumar, S., Khan, S. A., \& Jain, V., 2016. Designing an integrated AHP based decision support system for supplier selection in automotive industry. Expert Systems with Applications, 62, 273-283. doi:10.1016/j.eswa.2016.06.030

[10] Erceg, Ž., \& Mularifović, F., 2019. Integrated MCDM model for processes optimization in the supply chain management in the wood company. Operational Research in Engineering Sciences: Theory and Applications, 2(1). doi:10.31181/oresta1901015e

[11] Govindan, K., Kadziński, M., \& Sivakumar, R., 2017. Application of a novel PROMETHEE-based method for construction of a group compromise ranking to prioritization of green suppliers in food supply chain. Omega, 71, 129-145. doi:10.1016/j.omega.2016.10.004

[12] Govindan, K., Khodaverdi, R., \& Jafarian, A., 2013. A fuzzy multi criteria approach for measuring sustainability performance of a supplier based on triple bottom line approach. Journal of Cleaner Production, 47, 345-354. doi:10.1016/j.jclepro.2012.04.014

[13] Gören, H. G., 2018. A decision framework for sustainable supplier selection and order allocation with lost sales. Journal of Cleaner Production, 183, 1156-1169. doi:10.1016/j.jclepro.2018.02.2

[14] Guo, X., Yuan, Z., \& Tian, B., 2009. Supplier selection based on hierarchical potential support vector machine. Expert Systems with Applications, 36(3), 6978-6985. doi:10.1016/j.eswa.2008.08.074
[15] Hu, K.-J., \& Yu, V. F., 2016. An integrated approach for the electronic contract manufacturer selection problem. Omega, 62, 68-81. doi:10.1016/j.omega.2015.08.010

[16] Kannan, D., Khodaverdi, R., Olfat, L., Jafarian, A., \& Diabat, A., 2013. Integrated fuzzy multi criteria decision making method and multiobjective programming approach for supplier selection and order allocation in a green supply chain. Journal of Cleaner Production, 47, 355-367. doi:10.1016/j.jclepro.2013.02.010

[17] Kumar, S., Kumar, S., \& Barman, A. G., 2018. Supplier selection using fuzzy TOPSIS multi criteria model for a small scale steel manufacturing unit. Procedia Computer Science, 133, 905-912.

[18] Lam, K.-C., Tao, R., \& Lam, M. C.-K., 2010. A material supplier selection model for property developers using Fuzzy Principal Component Analysis. Automation in Construction, 19(5), 608-618. doi:10.1016/j.autcon.2010.02.007

[19] Liao, C.-N., \& Kao, H.-P., 2010. Supplier selection model using Taguchi loss function, analytical hierarchy process and multi-choice goal programming. Computers \& Industrial Engineering, 58(4), 571 577. doi:10.1016/j.cie.2009.12.004

[20] Liao, C.-N., \& Kao, H.-P., 2011. An integrated fuzzy TOPSIS and MCGP approach to supplier selection in supply chain management. Expert Systems with Applications, 38(9), 10803-10811. doi:10.1016/j.eswa.2011.02.031

[21] Lin, Y.-T., Lin, C.-L., Yu, H.-C., \& Tzeng, G.-H., 2010. A novel hybrid MCDM approach for outsourcing vendor selection: a case study for a semiconductor company in Taiwan. Expert Systems with Applications, 37(7), 4796-4804. doi:10.1016/j.eswa.2009.12.036

[22] Liu, Y., Eckert, C., Yannou-Le Bris, G., \& Petit, G., 2019. A fuzzy decision tool to evaluate the sustainable performance of suppliers in an agrifood value chain. Computers \& Industrial Engineering, 127, 196212. doi:10.1016/j.cie.2018.12.022

[23] Matić, B., Jovanović, S., Das, D. K., Zavadskas, E. K., Stević, Ž., Sremac, S., \& Marinković, M., 2019. A new hybrid mcdm model: sustainable supplier selection in a construction company. Symmetry, 11(3). doi:10.3390/sym11030353

[24] Mubarik, M. S., Kazmi, S. H. A., \& Zaman, S. I., 2021. Application of gray DEMATEL-ANP in green-strategic sourcing. Technology in Society, 64. doi:10.1016/j.techsoc.2020.101524

[25] Nielsen, I. E., Banaeian, N., Golińska, P., Mobli, H., \& Omid, M., 2014. Green Supplier Selection Criteria: From a Literature Review to a Flexible

[26] Pamučar, D., \& Ecer, F., 2020. Prioritizing the weights of the evaluation criteria under fuzziness: the fuzzy full consistency method - FUCOM-F. Facta Universitatis, Series: Mechanical Engineering, 18(3). doi:10.22190/fume200602034p

[27] Pamučar, D., Deveci, M., Canitez, F., \& Božanić, D., 2020. A fuzzy Full Consistency Method-Dombi-Bonferroni model for prioritizing transportation demand management measures. Applied Soft Computing, 87. doi:10.1016/j.asoc.2019.105952

[28] Pamučar, D., Stević, Ž., \& Sremac, S., 2018. A new model for determining weight coefficients of criteria in MCDM models: full consistency method (FUCOM). Symmetry, 10(9). doi:10.3390/sym10090393

[29] Rezaei, J., Fahim, P. B. M., \& Tavasszy, L., 2014. Supplier selection in the airline retail industry using a funnel methodology: Conjunctive screening method and fuzzy AHP. Expert Systems with Applications, 41(18), 8165-8179. doi:10.1016/j.eswa.2014.07.005

[30] Rouyendegh, B. D., \& Saputro, T. E., 2014. Supplier selection using integrated fuzzy TOPSIS and MCGP: a case study. Procedia - Social and Behavioral Sciences, 116, 3957-3970. doi:10.1016/j.sbspro.2014.01.874

[31] Stević, Ž., Durmić, E., Gajić, M., Pamučar, D., \& Puška, A., 2019. a novel multi-criteria decision-making model: interval rough SAW method for sustainable supplier selection. Information, 10(10). doi:10.3390/info10100292

[32] Sureeyatanapas, P., Sriwattananusart, K., Niyamosoth, T., Sessomboon, W., \& Arunyanart, S., 2018. Supplier selection towards uncertain and unavailable information: an extension of TOPSIS method. Operations Research Perspectives, 5, 69-79. doi:10.1016/j.orp.2018.01.005

[33] Taşkent, M.C., (2021). Tedarikçi Seçimi İçin Kriter Ağırlıklarının Eniyilenmesi Temelli Yeni Bir Bulanık Çok Kriterli Karar Verme Yaklaşımı. Yüksek Lisans Tezi, Atatürk Üniversitesi Fen Bilimleri Enstitüsü, Erzurum. 